\title{
Sperm maturation process occurs in the seminal vesicle following sperm transition from testis in honey bee males
}

\author{
Shinya HaYashi ${ }^{1}$, Toshiyuki SatoH ${ }^{2}$ \\ ${ }^{1}$ The United Graduate School of Agriculture, Tokyo University of Agriculture and Technology, 3-5-8 Saiwai-cho, Fuchu, \\ Tokyo, Japan \\ ${ }^{2}$ Department of Veterinary Medicine, Institute of Agriculture, Tokyo University of Agriculture and Technology, 3-5-8 \\ Saiwai-cho, Fuchu, Tokyo, Japan
}

Received 25 April 2018 - Revised 14 March 2019 - Accepted 26 April 2019

\begin{abstract}
In sexual reproduction, ejaculate components that males transfer into the females vary because of various factors. For male honey bees, the entirety of their sperm is stored in the seminal vesicles until the first copulation with a female and subsequent ejaculation, after which the male will die. Therefore, we can evaluate agerelated ejaculate alterations by investigating internal sexual organ changes with age. This study found that seminal vesicle wet weight decreased following sperm transfer from testes to seminal vesicles, while mucus gland weight was unchanged, thus resulting in increased sperm density in the seminal vesicles. This suggests that the decrease of seminal fluid in the seminal vesicles may be the underlying cause for age-related ejaculate component alterations.
\end{abstract}

drone / flight / seminal vesicle / sexual maturation / sperm density

\section{INTRODUCTION}

In sexual reproduction, the reproductive success of males depends on the number of eggs they can fertilise, which is restricted by the frequency of encounters with females and the number of sperm the males are capable of transmitting to the females (Bateman 1948; Trivers 1972; Parker 1984). Males transfer sperm and seminal fluid as ejaculate into females. These ejaculate components vary with the mating systems and

Electronic supplementary material The online version of this article (https://doi.org/10.1007/s13592-019-00652-5) contains supplementary material, which is available to authorized users.

Corresponding author: S. Hayashi, drone.honeybee@gmail.com Handling editor: Yves Le Conte operating sex ratio of focal species (Simmons 2001). In particular, males in polyandrous mating systems are exposed to severe intra-sexual selection, represented by sperm competition and cryptic female choice during egg fertilisation, leading to male evolutionary adaptations related to the enhancement of egg fertilisation, such as increasing the number of sperm, increased sperm motility, and elevated seminal fluid production (Parker 1970; Eberhard 1996; Simmons 2001; den Boer et al. 2010; Pearcy et al. 2014). Sperm quality and seminal fluid volume are important indicators for male fitness, but ejaculate composition and fertilisation capability change with ageing and the growing environment (Radwan 2003; Rhodes et al. 2011; Stürup et al. 2013; Simmons et al. 2014; Ruhmann et al. 2016). Therefore, determining what causes the changes in ejaculate components over time would allow us to better 
understand the causes of fertilisation and maturation.

A typical honey bee (Apis mellifera) colony consists of a queen and several thousand workers. During the reproduction season, several hundred males (drones) are produced. Drones initiate flight at about 8 days after emergence and take mating flights after sexual maturity a few days later (Howell and Usinger 1933; Ruttner 1966; Rueppell et al. 2006). Mature drones aggregate at specific mating places called "drone congregation areas" (DCA) in order to mate with females. Honey bees have an extremely male-biased operating sex ratio, with about 20,000 males present per female (Page and Metcalf 1984), meaning they are exposed to intense competition for mating. The females visiting DCA will mate with more than 10 males on average (Koeniger and Koeniger 2000; Tarpy et al. 2004; Kraus et al. 2005; El-Niweiri and Moritz 2010). Recent research has shown that females mate with over 50 males, much more than previously realised (Withrow and Tarpy 2018). This higher mating frequency reduces the potential effects of inbreeding at the colony level. Minimising the mating frequency among relatives is important because of their complementary sex determination system (Cook 1993; Zayed et al. 2005). In this sex determination system, inbreeding increases production of homozygous diploid drones which are consumed by workers in the early larval stage, result in spotty brood and reduction in colony fitness (Hedrick et al. 2006). Furthermore, such a high mating frequency indirectly enhances colony fitness through an increase in genetic diversity within the colony (Mattila and Seeley 2007). However, most of the sperm that transfers from a drone to a female is expelled through the vagina of the female during the sperm storage process. This results in only $3-5 \%$ of the sperm provided to the spermatheca being utilised for fertilisation over the life of the bee (Woyke 1983; Baer 2005). Such conditions drive males to maximise the amount of sperm and seminal fluid transferred into a female given the low probability of mating and the presence of male-competition after mating via sperm competition (Schlüns et al. 2003; Boomsma et al. 2005; den Boer et al. 2010). Honey bee drones show a remarkable characteristic: they die immediately after ejaculation (Ruttner 1966; Winston 1987; Koeniger and Koeniger 2000; Baer 2005).

Drones transfer sperm, seminal fluid, and mucus into the bulb of the everted endophallus and subsequently ejaculate semen into the female's oviduct (Woyke 2008). Mucus remains in the bulb of the endophallus. This helps to maintain the connection between a flying female and the drone until semen transfer into the female's oviducts is completed, which left in the sting chamber as a mating sign following ejaculation (Koeniger and Koeniger 2000; Woyke 2008). These components are produced by the sexual organs, composed of the testes, seminal vesicles, and mucus glands. Sperm produced in the testes moves to the seminal vesicles from 3 days after emergence until 8 days after emergence (Bishop 1920; Kurennoi 1953; Jaycox 1961). These sperm are stored with seminal fluid produced in seminal vesicles until ejaculation (Collins et al. 2006). The mucus glands fill with secretions consisting of mucus and seminal fluid up to 6 days after emergence (Colonello and Hartfelder 2003). During this timeframe, the ejaculate is considered to have no fertilisation capabilities, requiring a few days to mature and acquire fertilisation potential (Bishop 1920; Ruttner 1966; Woyke and Jasinski 1978). This timeline is consistent with the timing of drone mating flight initiation. However, the semen maturation process after the transition of sperm from the testes to seminal vesicles remains unclear. Drones transfer all of ejaculate components consisting of sperm and seminal fluid into the female's oviduct (Woyke 2008), allowing us to reveal the semen maturation process and the changes to ejaculate components related to egg fertilisation through evaluating the sexual organs after the transition of sperm from the testes. In this study, we measured sperm counts in the testes and seminal vesicles, as well as the wet weights of the testes, seminal vesicles, and mucus glands at multiple time points. In addition, we investigated the effect of flight development on sexual maturation by comparing drones with the opportunity to fly - i.e. having initiated flight before reaching sexual maturity - with those without (Howell and Usinger 1933; Ruttner 1966; Rueppell et al. 2006). 


\section{MATERIALS AND METHODS}

\subsection{Drone collection}

This study used colonies of a European honey bee subspecies, A. mellifera ligustica, (Kumagaya Apiary Co. Ltd., Saitama, Japan), which were reared at the Tokyo University of Agriculture and Technology, Fuchu, Tokyo, Japan. The study was conducted between July and early August 2012. In order to obtain many drones simultaneously, empty drone combs were introduced into queenright colonies possessing 20,000-32,000 workers. Eggs were laid in these drone combs by queens from two different colonies. The drone combs were removed from colonies 3 days prior to drone emergence and kept in an incubator at $33{ }^{\circ} \mathrm{C}$ until emergence. In order to identify the age of individual drones (day after emergence), emerged drones were marked with a paint marker (Mitsubishi Co. Ltd., Tokyo, Japan) on the thorax and abdomen within 24-h post-emergence.

To evaluate the effect of flight on internal sexual organ maturation, we prepared both drones which were allowed to fly freely (control) and drones which were prevented from flying (big back) via the attachment of a $0.5-\mathrm{mm}$ bead on the thorax using instant adhesive (Touagousei Co. Ltd., Tokyo, Japan). Individual drones in the control group were only treated on the thorax with instant adhesive. Hive entrances were narrowed by attaching fences, thus ensuring that control drones were able to pass through the entrance, but bead-attached drones could not. Both groups of drones were introduced into a foster colony and were kept there until sampling. Sampling was conducted in the early morning to avoid the peak drone flight time. We collected control and big back drones at $6,8,10$, and 14 days of age ( 6 days of age: $n=29,28 ; 8$ days of age: $n=$ 37,$34 ; 10$ days of age: $n=33,36 ; 14$ days of age: $n=27,30$ for control and big back groups, respectively).

\subsection{Sexual organ dissection and weighing}

The abdomens of collected drones were separated from the body with dissection scissors. After 5 min - as immediate abdominal dissection frequently induces ejaculation - the abdomen was dissected, with the testes, seminal vesicles, and mucus glands removed using scissors and tweezers. The abdomen was treated with $0.9 \% \mathrm{NaCl}$ throughout the procedure. Each sexual organ was put on a glass slide, with excess water removed using a delicate task wipe through capillary action, after which wet weight was measured. Subsequently, the number of spermatozoa within the testes and seminal vesicles was measured.

\subsection{Spermatozoa count measurements}

Pairs of testis or seminal vesicles were individually transferred into individual petri dishes (diameters $30 \mathrm{~mm}$ and $45 \mathrm{~mm}$ for testis and seminal vesicles, respectively) and incubated in $1 \mathrm{ml}$ of $0.9 \% \mathrm{NaCl}$ solution. Tissue was then torn with tweezers in order to release and disperse sperm within the $\mathrm{NaCl}$ solution. The solution was then diluted with tap water up to a total volume of $2 \mathrm{ml}$ for testes and $10 \mathrm{ml}$ for seminal vesicles. The diluted solutions containing spermatozoa were gently stirred with tweezers, following which spermatozoa were counted using a Thoma counting chamber under a light microscope. The number of spermatozoa was estimated by counting eight replicate fields.

\subsection{Statistical analyses}

To analyse age-related and flight treatmentrelated differences in the weights of testes, seminal vesicles, and mucus glands, we used a generalised linear mixed model (GLMM) with a gamma distribution. The weight of each sexual organ was treated as the response variable, while age and flight treatment were treated as explanatory variables. The source colony ID of drones was specified as random effect.

To analyse differences in testis and seminal vesicle sperm counts with age or flight treatment, we used a GLMM with a negative binomial distribution because of over dispersion. The number of sperm in each testis and seminal vesicle was treated as the response variable, while age and flight treatment were treated as explanatory variables. The source colony ID of drones was specified as random effect. Quadratic terms were also included in the statistical modelling to explain non-linear data. 
Furthermore, we analysed the number of sperm per milligram of seminal vesicle (sperm density) for each age group using a GLMM with a negative binomial distribution. The number of sperm in the seminal vesicle was treated as the response variable, age was treated as the explanatory variable, and seminal vesicle weight was treated as the offset term. The source colony ID of drones was specified as random effect. Quadratic terms were also included in the statistic modelling to explain non-linear data.

If significant differences were identified using the analysis methods listed above, we then performed multiple comparisons between ages using a GLMM, with $P$ values corrected using the Bonferroni method. We used R 3.4.3 with the Lme4 package to perform the GLMM analysis with a gamma distribution and aod package to perform the GLMM analysis with a negative binomial distribution. $P$ values were calculated using likelihood ratio tests.

\section{RESULTS}

Sexual organ weights and spermatozoa counts in the testes and seminal vesicles of control and big back drones were not significantly different (GLMM for sexual organ weights: $d f=1, \chi^{2}=$ 0.196, $P=0.658 ; d f=1, \chi^{2}=0.196, P=0.465$; $d f=1, \chi^{2}=0.020, P=0.888$ for testes, seminal vesicles, and mucus glands, respectively; GLMM for spermatozoa counts: $d f=1, \chi^{2}=0.093, P=$ $0.761 ; d f=1, \chi^{2}=95.637, P=0.186$ for testes and seminal vesicles, respectively). Therefore, all subsequent analyses presented here were conducted in the control group. Big back group results can be found in the Supplementary Table.

Age was found to have a significant effect on testis and seminal vesicle weight (GLMM: age: $d f$ $=1, \chi^{2}=509.450, P<0.001 ; d f=1, \chi^{2}=95.637$, $P<0.001$ for testis and seminal vesicle, respectively; see Supplemental Table for full details). Testis and seminal vesicle weight decreased significantly with age (Figure 1a, b). Age did not have a significant effect on mucus gland weight from 6 days post-emergence (GLMM: age: $d f=1, \chi^{2}=$ $0.5343, P=0.465$, Figure 1c).

Age had a significant effect on testis and seminal vesicle spermatozoa numbers (GLMM: age: $d f=1, \chi^{2}=11.170, P<0.001 ;$ GLMM: age: $d f$
$=1, \chi^{2}=22.680, P<0.001$, see the Supplemental Table for full details), but no significant difference was found across ages for testis and seminal vesicle, respectively (Figure 2a, b). This indicates that the transition of sperm from testis to seminal vesicle had almost concluded by $6-8$ days postemergence (Figure 2a, b). We also analysed changes in seminal vesicle sperm density with age. Sperm density significantly increased with age and reached maximum levels at about 10 days after emergence (GLMM: age: $d f=1, \chi^{2}=$ 28.620, $P<0.001$, Figure 3).

\section{DISCUSSION}

In the current study, we investigated the changes in the composition of semen in sexual organs with age, after transition of sperm from testis to seminal vesicle and production of mucus. This was done with the goal of revealing the maturation process of semen in the sexual organs and identifying a direct cause for age-related changes in ejaculate components early in life. Testis weight decreased with age, reflecting the transfer of sperm from the testes to the seminal vesicles (Figures 1a and 2a). Seminal vesicle spermatozoa numbers increased with age, but seminal vesicle weight decreased with age (Figures $1 \mathrm{~b}$ and $2 \mathrm{~b}$ ). Seminal vesicle sperm density increased following the completion of sperm testis-seminal vesicle transfer. Additionally, we also investigated the effect of flight on sexual maturation by comparing drones which had the opportunity to fly and those prevented from doing so. Flight did not confer a significant effect on sexual maturation. These results suggest that sperm or seminal fluid matures with age in the seminal vesicle though a process that increases sperm density and changes ejaculate components with age.

Ejaculate or sperm composition changes with age have been reported in mammals, including primates, but little else is known about this phenomenon, especially with regard to fertilisation in insects (Radwan 2003; Collins et al. 2006). Honey bee drones produce sperm in the testes during the pupal life stage and initiate testis-seminal vesicle transition of sperm after emergence (Bishop 1920). Currently, the prevailing belief is that sperm acquires the ability to fertilise eggs while in the seminal vesicle, but the process has 
a

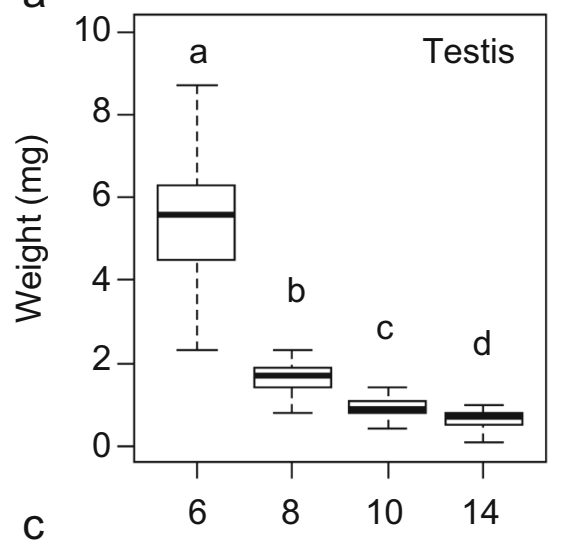

C

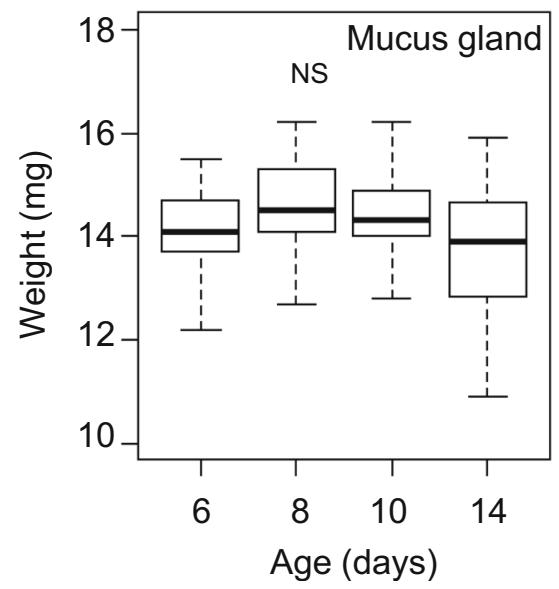

b

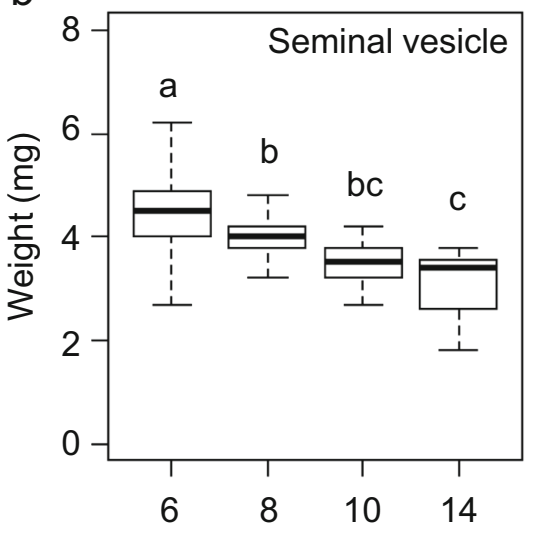

Figure 1 Wet weight (mean \pm SE) of testes (a), seminal vesicles (b), and mucus glands (c) for each age group in control bees. Statistical differences determined using GLMM between age groups are represented with different letters on the graph.

remained unclear. In this study, we found that seminal vesicle weight decreased but sperm density increased after the completion of the sperm transition from the testis to the seminal vesicle. This indicates that the increase in sperm density resulted from a decrease in non-sperm substances, such as haemolymph, after sperm transition had completed. Some previous studies have reported decreasing semen volume included in ejaculate, increasing viscosity, and changing from light cream to dark cream colour of semen with age, which suggests an increase in sperm concentration in the ejaculate. These trends correspond to an increased chance of successful fertilisation (Woyke and Jasinski 1978; Locke and Peng 1993; Cobey 2007; Czekońska et al. 2013). In addition, the tendency to alter ejaculates differed with season and colony (Rhodes et al. 2011; Rousseau et al. 2015). Our results regarding the role of the seminal vesicle may be explained by both increasing sperm concentration and decreasing ejaculate volume. Thus, it would reflect the importance of alterations to maturation or fertilisation capability. Furthermore, previous research has shown that the amount of semen remaining in seminal vesicles after ejaculation is higher in younger drones (Rhodes 2008), which would also explain the increase in sperm concentration in ejaculate with age. We consider that these changes are the cause of alterations to the ejaculate and represent their maturation process. The drones used in our study were relatively 

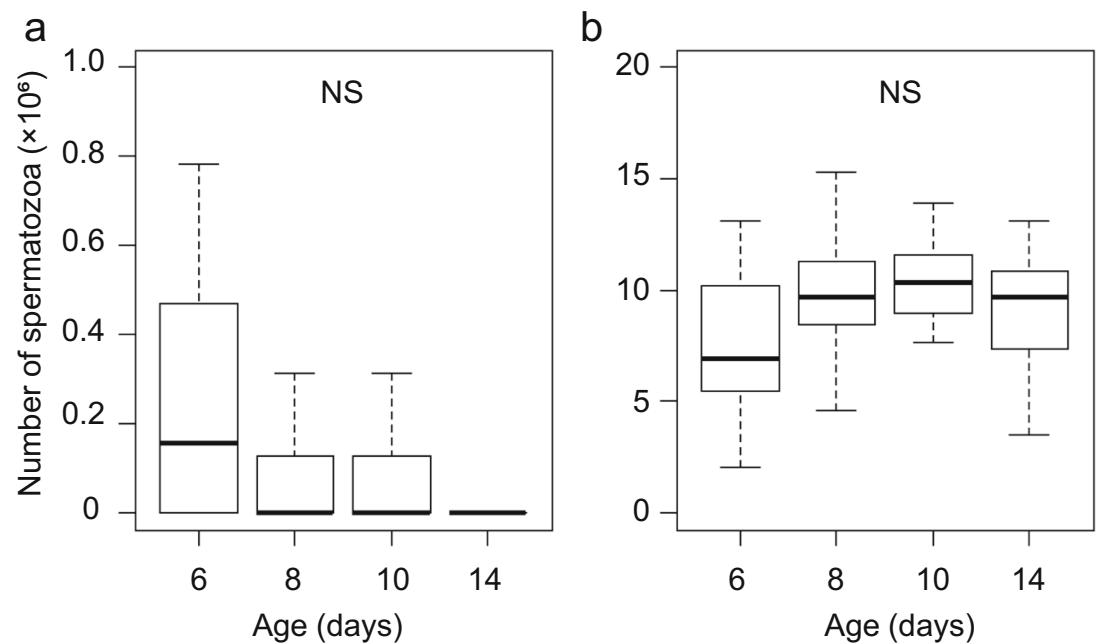

Figure 2 The number of spermatozoa (mean \pm SE) in testes (a) and seminal vesicles (b) for each age group in control bees.

young (6-14 days), and their maturation process may not proceed until after this period of their life (Rousseau et al. 2015). Our results do suggest a continuing maturation process in seminal vesicles following the transition of sperm from testes and may reflect sperm quality at a particular time.

Most social Hymenoptera males do not produce additional sperm in the testes upon reaching

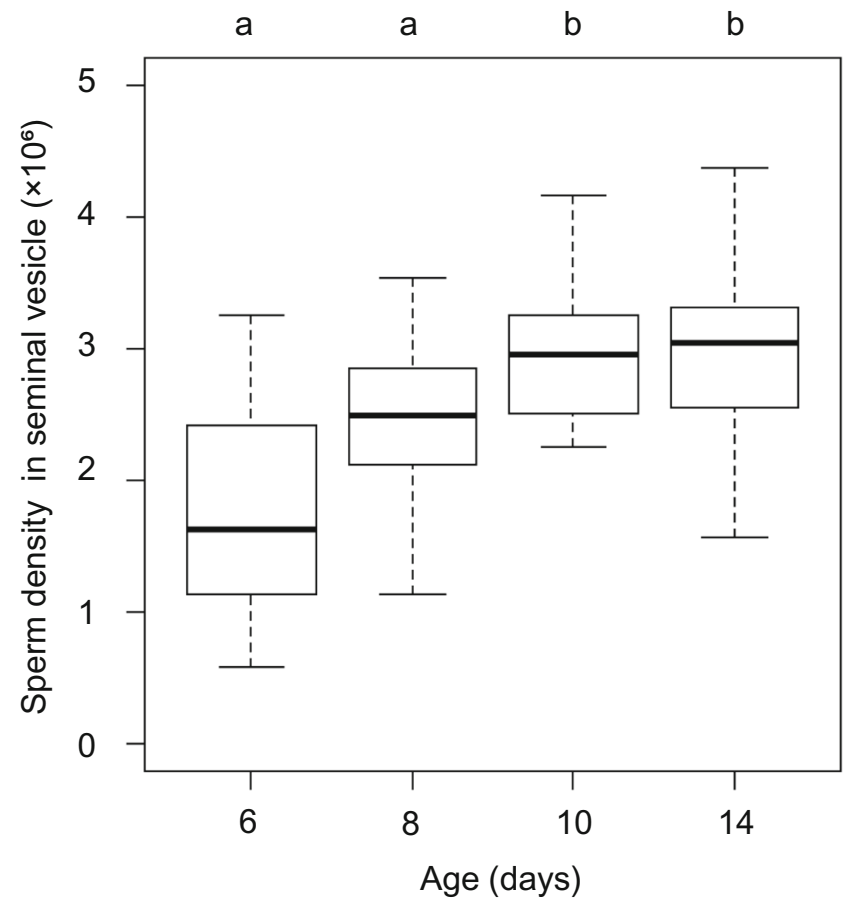

Figure 3 Sperm density (mean $\pm \mathrm{SE}$ ), calculated as the number of spermatozoa divided by the seminal vesicle wet weight, for each age group in control bees. Statistical differences determined using GLMM between age groups are represented with different letters on the graph. 
sexual maturity, and produced sperm are stored and maintained in the seminal vesicles (Bishop 1920; Hölldobler and Wilson 1990; Wheeler and Krutzsch 1992). This means that males must maintain sperm quality, such as viability and motility, in the seminal vesicle until mating. They are exposed to sperm competition after mating because the honey bee practices extreme polyandry (den Boer et al. 2010), meaning that maintaining viable high-quality sperm is a significant factor to increasing reproductive success in intra-sexual competition. Maintaining sperm viability is also important for females because their available sperm stock is determined by mating early in their adult life, which will ultimately determine their reproductive potential (Baer 2005). The epithelial cells lining the seminal vesicles produce seminal fluid, which comprises about $50 \%$ of the total semen volume (Verma and Shuel 1973). Seminal fluid proteins have functions related to metabolism, suggesting that they may contribute to sustaining sperm quality prior to and after mating (Collins et al. 2006; Baer et al. 2009). In this study, we suggested that the age-related seminal fluid decreases, and it may cause significant physiological changes related to the promotion and maintenance of higher sperm quality.

In post-mating competition, seminal fluid derived from mucus glands negatively affects sperm viability of competitor in the female reproductive duct, thus increasing the chance of reproductive success of the male (Baer et al. 2009; den Boer et al. 2010; Baer et al. 2012). In addition to this, seminal fluid also maintains sperm viability following ejaculation (den Boer et al. 2009; King et al. 2011). In this study, we could not identify any age-related changes in the mucus glands because they are usually filled with these secretions up to 6 days after emergence. However, previous studies have reported changes in mucus gland morphology, protein volume, and composition after the mucus glands are filled with seminal fluid and mucus (Colonello and Hartfelder 2003; Moors et al. 2005; Colonello-Frattini and Hartfelder 2009). Thus, these age-related changes may also affect male reproductive success by affecting sperm competition and postmating fertilisation.
We found that the timing of the completion of sperm transition from the testis to the seminal vesicle corresponded to the age of drone flight initiation, which was consistent with the results of Jaycox (1961). In addition, we found that increasing seminal vesicle sperm densities were not influenced by flight. Theses alterations that occur in sexual organs are probably age dependent. However, flight may decrease their potential benefits to fecundity in response to other vital function such as immunity and temperature resistance because maintaining sperm viability has a metabolic cost (Baer et al. 2006; Stürup et al. 2013). In insects, flight is the most intense activity, so that drones are likely to undergo oxidative stress during flight (Williams et al. 2008; Li-Byarlay et al. 2016), which might cause the reduction in sperm viability. Indeed, drones over 21 days after emergence experience a decrease in sperm viability (Locke and Peng 1993; Baer et al. 2012; Stürup et al. 2013); until that time they accumulate much flight time. Similarly, flight deprivation and an absence of defecation during their development by confinement might also affect sperm quality and mortality (Rhodes et al. 2011; Rousseau et al. 2015). However, there are few empirical studies that have evaluated the effect of flight or confinement on sperm quality, as most researchers have conducted confinement studies to investigate the alterations to ejaculation. Thus, evaluating the effects of flight on sperm quality should be focused on in future studies.

Most studies have reported shorter flight durations in honey bees when they are immature (Bishop 1920; Winston 1987; Fahrbach et al. 1997), suggesting the motivation behind flight is not related to mating in younger honey bees. Recently, a study reported that drones may memorise environmental features around the hive via flight experiences in order to return to their hive (Hayashi et al. 2017). As such, these pre-sexual maturity flights likely relate to hive orientation, similar to naïve worker honey bees (Capaldi and Dyer 1999). This information may be utilised to find and reach DCAs.

This study showed that sperm density increased in seminal vesicles after testis-seminal vesicle sperm transition, suggesting that agerelated ejaculate composition changes may be caused by decreasing seminal fluid volumes. 
These changes may increase individual chances of reproductive success in a polyandrous mating system with regard to maintaining viable and effective sperm.

\section{ACKNOWLEDGEMENTS}

Our thanks to Dr. Satoshi Koyama for useful advice and Prof. Ken-ichi Harano for honey bee keeping instruction. We sincerely thank Dr. Yuya Fukano for grateful advice regarding statistical analysis.

\section{AUTHOR'S CONTRIBUTIONS}

Shinya Hayashi conceived this research and designed experiments. S. Hayashi performed experiments and analysis, and wrote the paper with Toshiyuki Satoh.

Le processus de maturation du sperme se produit dans la vésicule séminale après la transmission du sperme par les testicules des abeilles mâles

Mâle / vol / vésicule séminale / maturation sexuelle / densité du sperme

Der Reifungsprozess der Spermien erfolgt in der Samenblase nach Spermienübertragung aus den Hoden der Drohnen

Drone / Flug / Samenblase / sexuelle Reifung / Spermiendichte

\section{REFERENCES}

Baer B (2005) Sexual selection in Apis bees. Apidologie $36: 187-200$.

Baer B, Armitage SAO, Boomsma JJ (2006) Sperm storage induces an immunity cost in ants. Nature 441:872-875.

Baer B, Heazlewood JL, Taylor NL, Eubel H, Millar AH (2009) The seminal fluid proteome of the honeybee Apis mellifera. Proteomics $9: 2085-2097$.

Baer B, Zareie R, Paynter E, Poland V, Millar AH (2012) Seminal fluid proteins differ in abundance between genetic lineages of honeybees. J. Proteome 75 :5646-5653.
Bateman AJ (1948) Intra-sexual selection in Drosophila. Heredity $2: 349-368$.

Bishop GH (1920) Fertilization in the honey-bee. I. The male sexual organs: their histological structure and physiological functioning. J. Exp. Zool. 31 :224-265.

Boomsma JJ, Baer B, Heinze J (2005) The evolution of male traits in social insects. Annu. Rev. Entomol. $\mathbf{5 0}: 395-420$.

Capaldi EA, Dyer FC (1999) The role of orientation flights on homing performance in honeybees. J. Exp. Biol. 202 : $1655-1666$.

Cobey SW (2007) Comparison studies of instrumentally inseminated and naturally mated honey bee queens and factors affecting their performance. Apidologie $38: 390-410$.

Collins AM, Caperna TJ, Williams V, Garrett WM, Evans JD (2006) Proteomic analyses of male contributions to honey bee sperm storage and mating. Insect Mol. Biol. $15: 541-549$.

Colonello NA, Hartfelder K (2003) Protein content and pattern during mucus gland maturation and its ecdysteroid control in honey bee drones. Apidologie $34: 257-267$.

Colonello-Frattini NA, Hartfelder K (2009) Differential gene expression profiling in mucus glands of honey bee (Apis mellifera) drones during sexual maturation. Apidologie $40: 481-495$.

Cook JM (1993) Hymenoptera: a review of models and evidence. Heredity (Edinb) $71: 421-435$.

Czekońska K, Chuda-Mickiewicz B, Chorbiński P (2013) The influence of honey bee (Apis mellifera) drone age on volume of semen and viability of spermatozoa. J. Apic. Sci. $57: 61-66$.

den Boer SPA, Boomsma JJ, Baer B (2009) Honey bee males and queens use glandular secretions to enhance sperm viability before and after storage. J. Insect Physiol. $55: 538-543$.

den Boer SPA, Baer B, Boomsma JJ (2010) Seminal fluid mediates ejaculate competition in social insects. Science 327 : 1506-1509.

Eberhard WG (1996) Female control : sexual selection by cryptic female choice. Princeton University Press, Princeton

El-Niweiri MAA, Moritz RFA (2010) The impact of apiculture on the genetic structure of wild honeybee populations (Apis mellifera) in Sudan. J. Insect Conserv. $14: 115-124$.

Fahrbach SE, Giray T, Farris SM, Robinson GE (1997) Expansion of the neuropil of the mushroom bodies in male honey bees is coincident with initiation of flight. Neurosci. Lett. 236 :135-138.

Hayashi S, Farkhary SI, Takata M, Satoh T, Koyama S (2017) Return of drones: flight experience improves returning performance in honeybee drones. J. Insect Behav. 30 :237-246. 
Hedrick PW, Gadau J, Page RE (2006) Genetic sex determination and extinction. Trends Ecol. Evol. 21 :55-57.

Hölldobler B, Wilson EO (1990) The ants. Harvard University Press, Cambridge

Howell DE, Usinger RL (1933) Observations on the flight and length of life of drone bees. Ann. Entomol. Soc. Am. $26: 239-246$.

Jaycox ER (1961) The effects of various foods and temperatures on sexual maturity of the drone honey bee (Apis mellifera). Ann. Entomol. Soc. Am. $54: 519-523$.

King M, Eubel H, Millar AH, Baer B (2011) Proteins within the seminal fluid are crucial to keep sperm viable in the honeybee Apis mellifera. J. Insect Physiol. 57 :409-414.

Koeniger N, Koeniger G (2000) Reproductive isolation among species of the genus Apis. Apidologie $31: 313-339$

Kraus FB, Neumann P, Moritz RFA (2005) Genetic variance of mating frequency in the honeybee (Apis mellifera L.). Insect. Soc. $52: 1-5$.

Kurennoi NM (1953) When are drones sexually mature. Pchelovodstvo $30: 28-32$.

Li-Byarlay H, Huang MH, Simone-Finstrom M, Strand MK, Tarpy DR, Rueppell O (2016) Honey bee (Apis mellifera) drones survive oxidative stress due to increased tolerance instead of avoidance or repair of oxidative damage. Exp. Gerontol. $83: 15-21$.

Locke SJ, Peng YS (1993) The effects of drone age, semen storage and contamination on semen quality in the honey bee (Apis mellifera). Physiol. Entomol. $18: 144-148$

Mattila HR, Seeley TD (2007) Genetic diversity in honey bee colonies enhances productivity and fitness. Science $317: 362-364$.

Moors L, Spaas O, Koeniger G, BIllen J (2005) Morphological and ultrastructural changes in the mucus glands of Apis mellifera drones during pupal development and sexual maturation. Apidologie $36: 245-254$.

Page REJ, Metcalf RA (1984) A population investment sex ratio for the honey bee (Apis mellifera L.). Am. Nat. $124: 680-702$.

Parker GA (1970) Sperm competition and its evolutionary consequences in the insects. Biol. Rev. 45 :525-567.

Parker GA (1984) Sperm competition and the evolution of animal mating strategies. Academic Press, Orlando

Pearcy M, Delescaille N, Lybaert P, Aron S (2014) Team swimming in ant spermatozoa. Biol. Lett. $10: 20140308-20140308$.

Radwan J (2003) Male age, germline mutations and the benefits of polyandry. Ecol. Lett. $6: 581-586$.

Rhodes JW (2008) Semen production in drone honeybees. Rural Industries Research and Development Corporation, Wagga Wagga
Rhodes JW, Harden S, Spooner-Hart R, Anderson DL, Wheen $\mathrm{G}$ (2011) Effects of age, season and genetics on semen and sperm production in Apis mellifera drones. Apidologie 42 :29-38.

Rousseau A, Fournier V, Giovenazzo P (2015) Apis mellifera (Hymenoptera: Apidae) drone sperm quality in relation to age, genetic line, and time of breeding. Can. Entomol. 147 :702-711.

Rueppell O, Page REJ, Fondrk MK (2006) Male behavioural maturation rate responds to selection on pollen hoarding in honeybees. Anim. Behav. $71: 227-234$.

Ruhmann H, Wensing KU, Neuhalfen N, Specker J-H, Fricke C (2016) Early reproductive success in Drosophila males is dependent on maturity of the accessory gland. Behav. Ecol. 27 :1859-1868.

Ruttner F (1966) The life and flight activity of drones. Bee World $47: 93-100$.

Schlüns H, Schlüns EA, van Praagh J, Moritz RFA (2003) Sperm numbers in drone honeybees ( Apis mellifera ) depend on body size. Apidologie $34: 577-584$.

Simmons LW (2001) Sperm competition and its evolutionary consequences in the insects. Princeton University Press, Princeton

Simmons LW, Beveridge M, Li L, Tan Y-F, Millar AH (2014) Ontogenetic changes in seminal fluid gene expression and the protein composition of cricket seminal fluid. Evol. Dev. 16 :101-109.

Stürup M, Baer-Imhoof B, Nash DR, Boomsma JJ, Baer B (2013) When every sperm counts: factors affecting male fertility in the honeybee Apis mellifera. Behav. Ecol. 24 :1192-1198.

Tarpy DR, Nielsen R, Nielsen DI (2004) A scientific note on the revised estimates of effective paternity frequency in Apis. Insect. Soc. 51 :203-204.

Trivers RL (1972) Parental investment and sexual selection. Sex. Sel. Descent Man (ed. Campbell B), London Heinemann 136-179.

Verma LR, Shuel RW (1973) Respiratory metabolism of the semen of the honey-bee, Apis mellifera. J. Insect Physiol. 19 :97-103.

Wheeler DE, Krutzsch PH (1992) Internal reproductive system in adult males of the genusCamponotus (Hymenoptera: Formicidae: Formicinae). J. Morphol. $211: 307-317$.

Williams JB, Roberts SP, Elekonich MM (2008) Age and natural etabolically-intensive behavior affect oxidative stress and antioxidant mechanisms. Exp. Gerontol. 43 :538-549.

Winston ML (1987) The biology of the honey bee. Harvard University Press, Cambridge

Withrow JM, Tarpy DR (2018) Cryptic "royal” subfamilies in honey bee (Apis mellifera) colonies. PLoS One $13: \mathrm{e} 0199124$. 
Woyke J (1983) Dynamics of entry of spermatozoa into the spermatheca of instrumentally inseminated queen honeybees. J. Apic. Res. $22: 150-154$.

Woyke J (2008) Why the eversion of the endophallus of honey bee drone stops at the partly everted stage and significance of this. Apidologie 39:627-636.

Woyke J, Jasinski Z (1978) Influence of age of drone son the results of instrumental insemination of honeybee queens. Apidologie 9 :203-212.
Zayed A, Packer L, Grixti JC, Ruz L, Owen RE, Toro H (2005) Increased genetic differentiation in a specialist versus a generalist bee: Implications for conservation. Conserv. Genet. $6: 1017-1026$.

Publisher's note Springer Nature remains neutral with regard to jurisdictional claims in published maps and institutional affiliations. 